\title{
A New Galactic Wolf-Rayet Star in Centaurus
}

\author{
A. Roman-Lopes \\ Astronomy Group, Department of Physics, Universidad de La Serena, Cisternas 1200, La Serena, Chile
}

Correspondence should be addressed to A. Roman-Lopes, roman@dfuls.cl

Received 6 April 2011; Accepted 16 May 2011

Academic Editor: J. A. L. Aguerri

Copyright (C) 2011 A. Roman-Lopes. This is an open access article distributed under the Creative Commons Attribution License, which permits unrestricted use, distribution, and reproduction in any medium, provided the original work is properly cited.

I communicate the detection of a new Galactic Wolf-Rayet star (WR60a) in Centaurus. The H- and K-band spectra of WR60a show strong carbon near-infrared emission lines, characteristic of Wolf-Rayet stars of the WC5-7 subtype. Adopting mean absolute magnitude $M_{K}$ and mean intrinsic $\left(J-K_{S}\right)$ and $\left(H-K_{S}\right)$ colours, it was found that WR60a suffers a mean visual extinction of $3.8 \pm 1.3$ magnitudes, being located at a probable heliocentric distance of $5.2 \pm 0.8 \mathrm{Kpc}$, which for the related Galactic longitude $(1=312)$ puts this star probably in the Carina-Sagittarius arm at about $5.9 \mathrm{kpc}$ from the Galactic center. I searched for clusters in the vicinity of WR60a and in principle found no previously known clusters in a search radius region of several tens arcminutes. The detection of a well-isolated WR star induced us to seek for some still unknown cluster, somewhere in the vicinity of WR60a. From inspection of $5.8 \mu \mathrm{m}$ and $8.0 \mu \mathrm{m}$ Spitzer/IRAC GLIMPSE images of the region around the new WR star, strong mid-infrared extended emission at about $13.5 \mathrm{arcmin}$ south-west of WR60a was found. The study of the $\mathrm{H}-\mathrm{K}_{S}$ colour distribution of point sources associated with the extended emission reveals the presence of a new Galactic cluster candidate probably formed by at least 85 stars.

\section{Introduction}

Despite the important role that WR stars probably play in shaping both Galactic structure and chemical evolution, the total number of WR stars in the Milk Way is still an open issue. Shara et al. [1] and Van Der Hucht. [2] estimate a number between 1000 and $2500 \mathrm{WR}$ stars to be located within the Galaxy, which compared to the about 300 known Galactic WR stars [3] indicates that, up to date, there still a large number of such stars to be discovered in our Galaxy. One reason for this comes from the fact that dust obscuration makes hard to observe WR stars in the optical window through the entire Galactic plane. On the other hand, infrared observations can provide the means for finding a significant fraction of the remaining population of obscured Galactic WR stars. Indeed, in the near-infrared (NIR) window, $H$ and $K$ band spectroscopy may be used to properly identify and classify the new Galactic WR stars, accordingly to the known WC, WN, and WO subtypes [4-6].

In this work, I report the discovery of a new Wolf-Rayet star in Centaurus through the detection of its NIR carbon and He emission lines, using ESO-NTT-SOFI archival spectroscopic data. In Section 2, I describe the observational data and the data reduction procedures, in Section 3, I present the results, and in Section 4, it is presented a summary of the work.

\section{Observational Data and Reduction Procedures}

2.1. Photometric Parameters of the Newly-Identified WR Star. Coordinates and photometric parameters for the new WR star are shown in Table 1. The near-IR photometric data were taken from the Two-Micron All Sky Survey 2MASS, [7] using the NASA/IPAC Infrared Science Archive (http://irsa.ipac.caltech.edu/applications/BabyGator/), while the $V$-band photometry was obtained from the Naval Observatory Merged Astronomical Dataset (NOMAD) (http://www.nofs.navy.mil/data/fchpix/vo_nofs.html). The star was named WR60a, following the common practice to giving numbers to Galactic WR stars in RA order, with 
TABLE 1: Some photometric parameters of the WR60a star. The near-IR photometric data were taken from the Two-Micron All Sky Survey (2MASS) using the NASA/IPAC Infrared Science Archive, while the $V$-band photometry was obtained from the Naval Observatory Merged Astronomical Dataset (NOMAD). For the derived spectral type, visual extinction, and heliocentric distance, see the text.

\begin{tabular}{|c|c|c|c|c|c|c|c|c|c|}
\hline ID & $\begin{array}{c}\text { RA } \\
(\mathrm{J} 2000)\end{array}$ & $\begin{array}{c}\text { Dec } \\
(\mathrm{J} 2000)\end{array}$ & V & $J$ & $H$ & $K_{s}$ & Sp type & $A_{V}(\mathrm{mag})$ & $d(\mathrm{Kpc})$ \\
\hline WR60a & 14:06:03.61 & $-60: 27: 29.5$ & 15.35 & 10.84 & 10.12 & 9.39 & WC5-7 & $3.8 \pm 1.3$ & $5.2 \pm 0.8$ \\
\hline
\end{tabular}

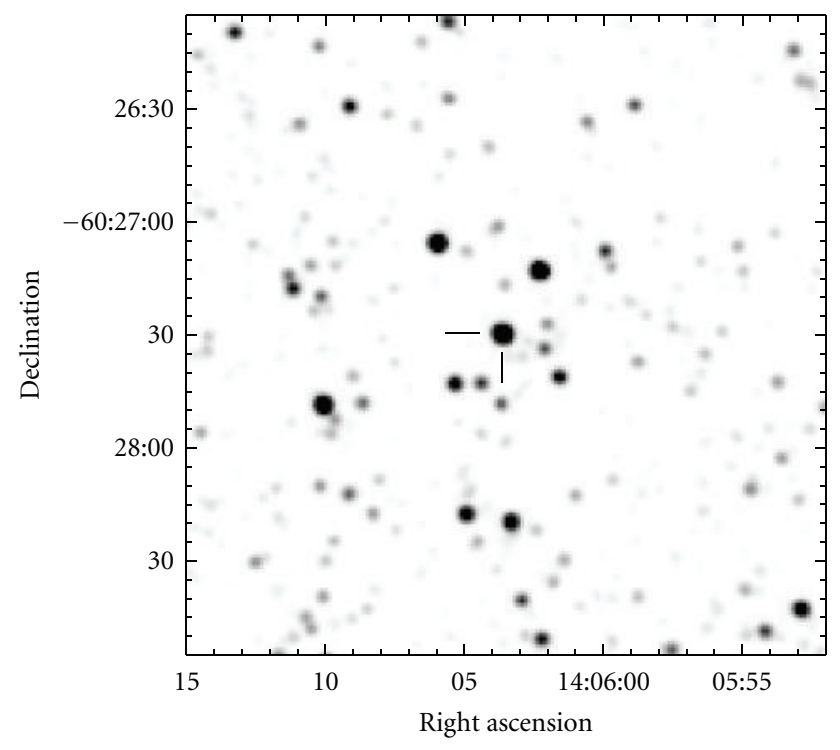

Figure 1: The $4 \operatorname{arcmin} \times 4$ arcmin 2 MASS $K_{S}$ band finding chart for the newlydiscovered Wolf-Rayet star WR60a. North is to the top, East to the left.

further additions between integers from Van Der Hucht $[2,3]$. A 2MASS $K_{S}$ band finding chart for this object is presented in Figure 1.

2.2. Near-Infrared Spectroscopic Observations. In this work, I used ESO archival data (http://archive.eso.org/eso/eso_ archive_main.html) obtained with the SofI instrument [8], coupled to the $3.6 \mathrm{~m} \mathrm{NTT}$ telescope. The spectra were taken as part of the ESO program 075.D-0210(A) (PI A.P. Marston and collaborators), with the targets being selected according to the technique presented by Hadfield et al. [9]. A summary of the spectroscopic dataset is presented in Table 2.

2.3. Data Reduction. The raw spectra were reduced following the NIR reduction procedures presented by Roman-Lopes [10], shortly described here. The two-dimensional frames were sky subtracted for each pair of images taken at the two nod positions $\mathrm{A}$ and $\mathrm{B}$, followed by division of the resultant image by a master flat. The multiple exposures were combined, followed by one-dimensional extraction of the spectra. Thereafter, wavelength calibration was applied using the sky lines; the typical error $(1-\sigma)$ for this calibration was $\sim 21 \AA$. Telluric atmospheric correction done using $H$ and $K$-band spectra of $\mathrm{A}$ and $\mathrm{B}$ type stars completed the reduction process.
TABLE 2: Summary of the NTT/SofI dataset used in this work.

\begin{tabular}{lc}
\hline Date & $2005-06-23$ \\
Telescope & NTT \\
Instrument & SofI \\
Grism & GR \\
Slit $(\operatorname{arcsec})$ & $0.6 \times 290$ \\
Resolution & 1000 \\
Coverage $(\mu \mathrm{m})$ & $1.53-2.52$ \\
Seeing $(\operatorname{arcsec})$ & $1-2$ \\
\hline
\end{tabular}

\section{Results}

3.1. The $H$ - and K-Band Spectra of WR60a. The $H$ - and $K$ band spectra of WR60a are shown in Figure 2, with the main helium and carbon emission lines identified by labels. From an inspection of the this figure, we can see that the carbon emission lines are very strong, with the most prominent being the $\mathrm{C}_{\mathrm{Iv}}$ line at $2.076 \mu \mathrm{m}$. On the other hand, from a comparison of the WR60a $K$-band spectrum with those from the spectral atlas of WRs stars of Figer et al. [11], we might conclude that this new WR star is probably of the WC5-7 subtype. Indeed, from the ratio (3.7) of the $\mathrm{C}_{\mathrm{IV}} 2.076 \mu \mathrm{m}$ and $\mathrm{C}_{\text {III }} 2.110 \mu \mathrm{m}$ equivalent widths (EWs), one can get the same conclusion [12].

3.1.1. A Probable Nondusty WC Star. Late-type WC stars sometimes are surrounded by circumstellar dust (which is believed to be produced by interaction with an $\mathrm{OB}$ binary companion), whose thermal emission may contribute to the underlying NIR continuum. As can be noticed in Figure 2, the $K$-band carbon lines of WR60a do not seem to suffer such effect in its underlying continuum. In one hand, the observed NIR colours of WR60a are compatible with that for a WC5-7 WR star $\left(\left(J-K_{S}\right)_{0}=0.62,\left(H-K_{S}\right)_{0}=0.58-[12]\right)$, reddened by $3.8 \pm 1.3 \mathrm{mag}$ in the $V$ band [13]. On the other hand, the absence of reduced line strengths in principle can not be used to discard the presence of a line-of-sight/binary early-type companion since relatively short period late-type WC binaries like $\gamma \mathrm{Vel}(\mathrm{WC} 8+\mathrm{O})$ are known not to form dust.

3.1.2. Heliocentric Distance to WR60a. Despite quantitative analysis of the newly discovered WR star is beyond the scope of this work, from the derived spectral type and associated NIR photometry, it is possible to obtain an estimate for the heliocentric distance to WR60a, considering the extinction law for NIR bands derived by Indebetouw et al. [13] and following the procedure described by Mauerhan et al. [6]. I used the mean values for absolute $K$-band magnitude 


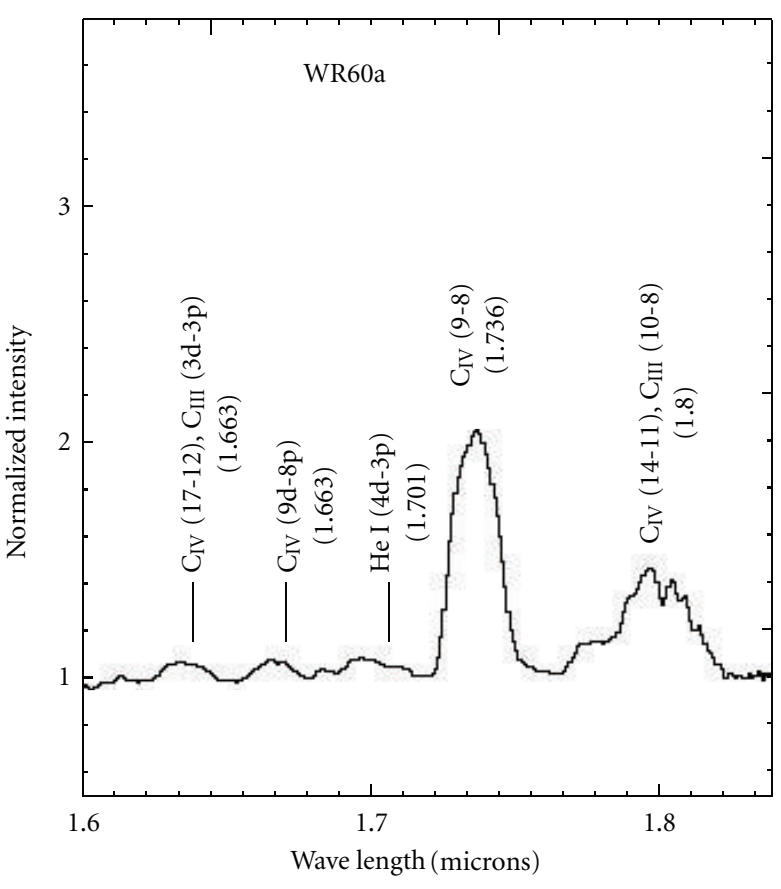

(a)

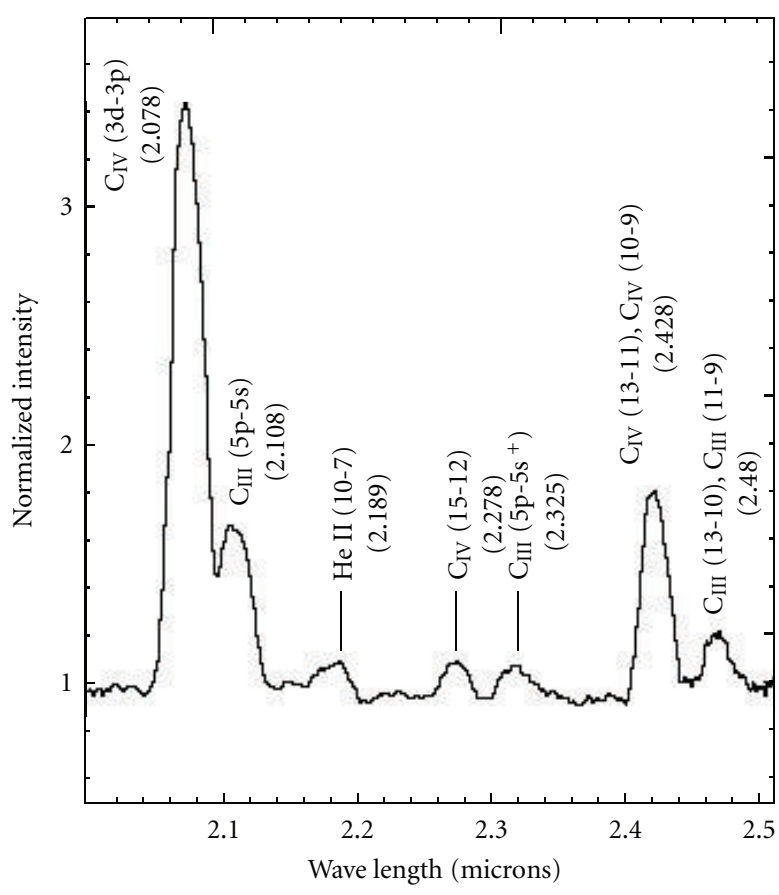

(b)

FIGURE 2: The continuum normalized $H$ - and $K$-band spectra of the new Wolf-Rayet star WR60a.

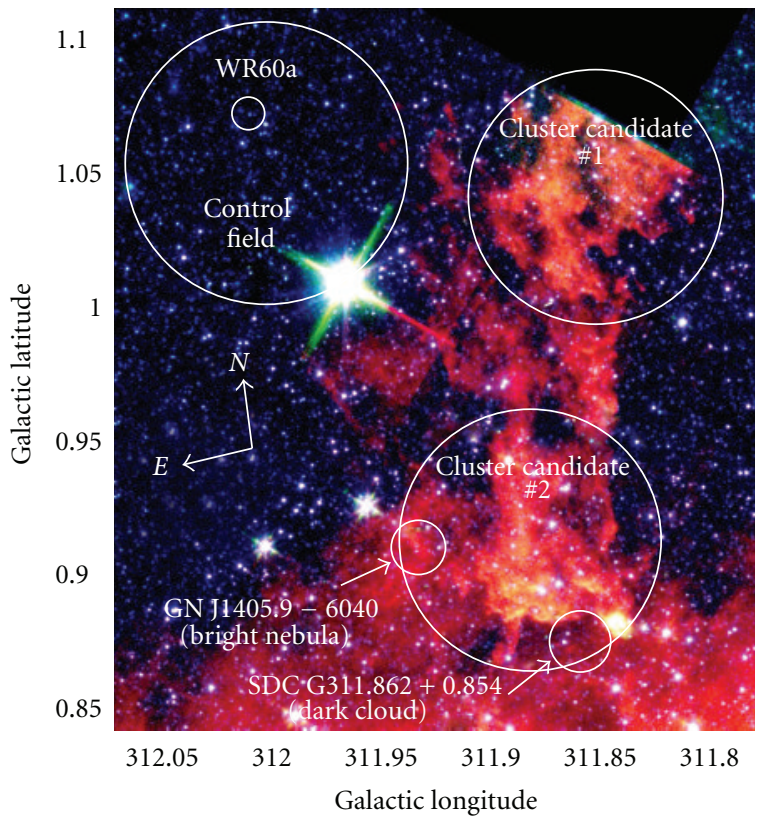

Figure 3: The Spitzer/IRAC $4.5 \mu \mathrm{m}$ (blue), $5.8 \mu \mathrm{m}$ (green), and $8.0 \mu \mathrm{m}$ (red) false-color image of the region around WR60a.

$\left(M_{K}=-4.59\right)$ and $J-K_{S}$ and $H-K_{S}$ colours $(0.62$ and 0.58 , resp.) taken from Crowther et al. [12]. It was found a mean heliocentric distance of $5.2 \pm 0.8 \mathrm{kpc}$, which puts this WR star at about $5.9 \mathrm{kpc}$ from the Galactic center, probably in the Carina-Sagittarius arm.
3.2. Cluster Candidates and Other WR Stars in the Vicinity of WR60a. I searched for cataloged clusters in the vicinity of WR60a and found no known clusters around it in a region of tens of arcminutes. In fact, the first known cluster is DBS2003 \#135 [14] with coordinates 14:08:42, $-61: 10: 36$ and located at about 47 arcmin from the new WR star. Also from a search in the clusters candidate list of Mercer et al. [15], the closest one is their number $\# 43$ ( $\alpha=14: 00: 28, \delta=-60: 59: 15-$ $J 2000$ ) that is located at about 52 arcmin from WR60a.

The presence of a well-isolated WR star naturally generates the question about the WR60a parental cluster candidate. In fact, the presence of such kind of star strongly suggests the existence of some nearby unknown stellar cluster. In order to address this question, I performed a visual search through 2MASS and Spitzer/IRAC images of the region towards the new WR star. I found no indication for the presence of clusters from a visual inspection of the 2MASS images. However, from the $5.8 \mu \mathrm{m}$ and $8.0 \mu \mathrm{m}$ Spitzer/IRAC images, it can be noticed the presence of strong extended emission, south-west of WR60a. In Figure 3, I present a false-color image made using the $4.5 \mu \mathrm{m}, 5.8 \mu \mathrm{m}$, and $8.0 \mu \mathrm{m}$ Spitzer/IRAC images of this Galactic sector, taken by the GLIMPSE survey. There, it is clearly seen the presence of several filamentary structures distributed perpendicularly to the Galactic plane and extending at least to $b \sim 1$.1. On the other hand, an inspection of the filamentary region indicates the existence of an apparent concentration of near- to mid-infrared point sources around the coordinates (J2000) 14:05:05.42, -60:31:22.9, and 14:05:35.07, $-60: 40: 30.5$ (hereafter named cluster candidate \#1 and cluster candidate \#2-see Figure 3), both located at angular 


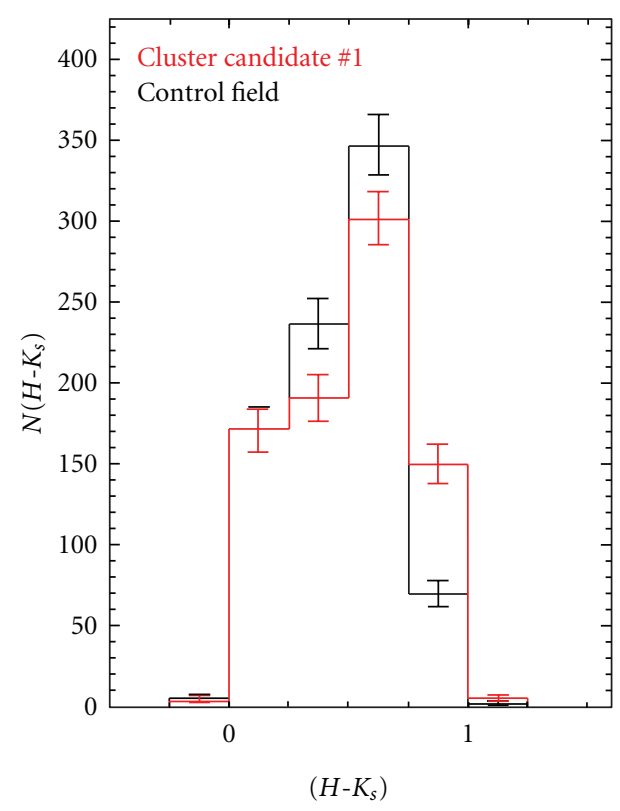

(a)

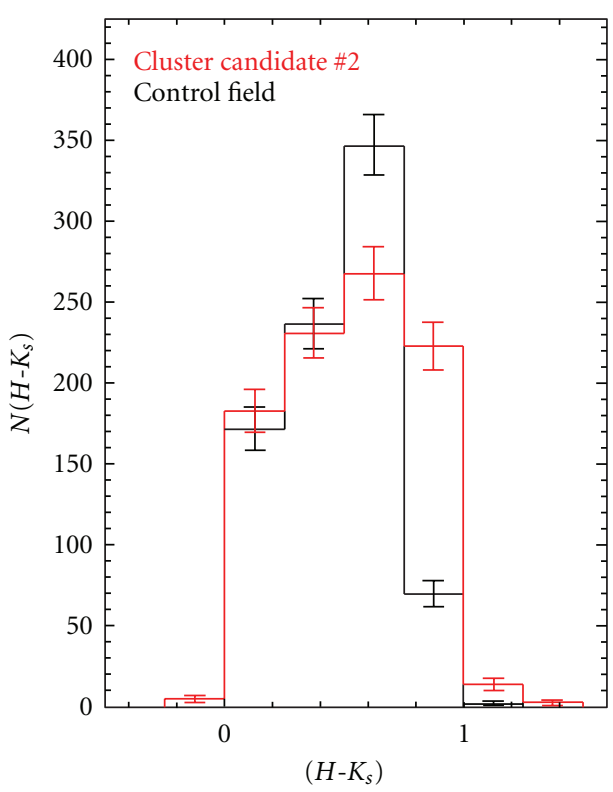

(b)

FIgURE 4: The $H-K_{S}$ colours histograms for the 2MASS sources detected in the direction of the two cluster candidates, together with that obtained from the control field (see Figure 3).

distances of 8 arcmin and $13.5 \mathrm{arcmin}$, respectively, from WR60a.

Finally, I also searched for other known Wolf-Rayet stars in the vicinity of WR60a; however, only two were found at angular distances greater than a degree! HD121194 (WC7) with coordinates 13:55:48.1, -61:09:50 (J2000) and SSTGLMCG3 $13.8558+00.6489$ (WN6) with coordinates 14:21:23.15, -60:18:04.1 (J2000).

3.2.1. Known Astrophysical Sources Possibly Associated to the Cluster Candidates \#1 and \#2. Using the SIMBAD Astronomical Database, I took a look in the literature for astrophysical sources around the central coordinates of both cluster candidates and found no sources related to the cluster candidate \#1. On the other hand, for the cluster candidate $\# 2$, there are two objects found at about 3 arcmin from its assigned coordinates. The first measuring about $1.7 \operatorname{arcmin} \times$ 1.1 arcmin is the bright nebula named GN J1405.9-6040 (14:05:58.53, -60:40:14.4 (J2000)), cataloged by kronberger et al. [16] from inspection of DSS-2 blue and red images. The second is the dark nebula SDC G311.862+0.854 (14:05:28.32, $-60: 43: 37$ (J2000)), which was cataloged by Peretto and Fuller [17] in a work aimed to identify Galactic infrared dark clouds (IRDCs), using Spitzer GLIMPSE and MIPSGAL data. The relative positions of this objects are indicated in Figure 3. It is interesting to notice that both nebula are placed at the border of the region assigned to the cluster candidate \#2, which may be indication of ongoing star formation within the host molecular clouds. However, only the detection of extended mid-infrared emission is not enough to properly support the assumption of the presence of a cluster of stars there. In the next section, it is presented some results that favor this assumption for the cluster candidate \#2.

3.2.2. NIR Colour Study of the Stellar Population Associated to the Cluster Candidates \#1 and \#2. The detailed study of the stellar population associated with the two cluster candidates identified in the previous section is out of the scope of this work. However, even a simple analyses of the NIR colour distributions can provide additional clues to support (or not) the idea of existence of clusters of stars associated with the two selected regions in Figure 3. In this sense, it was analyzed the $H-K_{S}$ colour distributions of point sources from three delimited areas, labeled cluster candidate \#1, cluster candidate \#2, and control field, the last centered at coordinates (J2000) 14:06:06.57, -60:28:46.2 and assumed to be mainly compound by Galactic field stars (with the obvious exception of WR60a). The study was done using the 2MASS H- and $K_{S}$-band photometry of sources present in the three selected regions, considering circular fields of $4 \mathrm{arcmin}$ radius (as illustrated in Figure 3 by the white large circles). The results are shown in Figure 4. From these diagrams, we can see that the stellar population in the direction of the cluster candidate \#1 is mainly formed by Galactic field stars. In fact, its $\mathrm{N}\left(H-K_{S}\right) \times H-K_{S}$ diagram presents an excess of field sources in the colour range $0.25 \leq H-K_{S} \leq 0.75(91 \pm 32)$ that is fully compensated by the excess of cluster \#1 sources in the range $0.75 \leq H-K_{S} \leq 1.0(81 \pm 14)$. On the other hand, the $N\left(H-K_{S}\right) \times H-K_{S}$ diagram for sources in the cluster candidate $\# 2$ region clearly indicates an excess of objects with $H-K_{S}$ colours greater than $0.75 \mathrm{mag}$. Indeed, the number of 
extra sources found in the control region in the colour range $0.5 \leq H-K_{s} \leq 0.75(80 \pm 25)$ is much less than that computed for the colour range $0.75 \leq H-K_{S} \leq 1.25(165 \pm 18)$. From this results, I can crudly estimate the number of probable members of the cluster candidate $\# 2$ at $85 \pm 31$. Considering that the photometric completeness of the 2MASS survey in the $H$ and $K_{S}$ bands restricts the analyses mainly to the more luminous sources, the true number of cluster members probably is greater than the inferred here. Of course, new further spectrophotometric studies are necessary to properly constrain the cluster population in this part of the Galaxy.

\section{Summary}

In this work, the detection of a new Galactic Wolf-Rayet star in the direction of Centaurus is reported. The $H$ - and $K$-band spectra of WR60a indicate that the star probably belongs to the WC5-7 subtype. From the derived spectral type and associated NIR photometry, it was computed a probable heliocentric distance of $5.2 \pm 0.8 \mathrm{Kpc}$, which for the related Galactic longitude $(l=312)$ puts this WR star at about $5.9 \mathrm{kpc}$ from the Galactic center, probably in the Carina-Sagittarius arm.

I searched for clusters in the vicinity of WR60a and in principle found no known cataloged clusters in a radius search region of several tens of arcminutes. On the other hand, I also searched for other known Wolf-Rayet stars in the vicinity of WR60a, with no detections inside a one-degree search radius!

The detection of a well-isolated WR star naturally leaved us to seek for some still unknown cluster, somewhere in the vicinity of WR60a. From visual inspection of $5.8 \mu \mathrm{m}$ and $8.0 \mu \mathrm{m}$ Spitzer/IRAC GLIMPSE images of the region around the new WR star, it was found strong mid-infrared extended emission produced by filamentary structures oriented perpendicularly to the Galactic plane. The study of the the $H-K_{S}$ colour distributions of point sources associated with the extended emission indicates the presence of a new Galactic cluster candidate (central coordinates (J2000) 14:05:35.07, $-60: 40: 30.5$ ) placed at about 13.5 arcmin southwest of WR60a, for which I estimate a lower limit of $85 \pm 31$ cluster members.

\section{Acknowledgments}

This work was partially supported by the ALMA-CONICYT Fund, under the project number 31060004, "A New Astronomer for the Astrophysics Group, Universidad de La Serena", by Dirección de Investigación Universidad de La Serena (DIULS) under program "Proyecto Convenio de Desempeño CD11103", and by the Department of Physics of the Universidad de La Serena." This publication makes use of data products from the Two-Micron All Sky Survey, which is a joint project of the University of Massachusetts and the Infrared Processing and Analysis Center/California Institute of Technology, funded by the National Aeronautics and Space Administration and the National Science Foundation.
This research has made use of the SIMBAD database, operated at CDS, Strasbourg, France. This work was based on observations made with ESO Telescopes at the La Silla Observatory under programme ID 075.D-0210(A).

\section{References}

[1] M. M. Shara, A. F. J. Moffat, L. F. Smith, V. S. Niemela, M. Potter, and R. Lamontagne, "A deep survey for galactic WolfRayet stars. II. Implications for galactic structure and massive star formation," Astronomical Journal, vol. 118, no. 1, pp. 390405, 1999.

[2] K. A. Van Der Hucht, "The VIIth catalogue of galactic WolfRayet stars," New Astronomy Reviews, vol. 45, no. 3, pp. 135232, 2001

[3] K. A. Van Der Hucht, "New Galactic Wolf-Rayet stars, and candidates (research note) an annex to the VIIth catalogue of galactic Wolf-Rayet stars," Astronomy and Astrophysics, vol. 458, no. 2, pp. 453-459, 2006.

[4] P. R. J. Eenens, P. M. Williams, and R. Wade, "An infrared view of Wolf-Rayet WC subtypes. I - Line identification and spectral characteristics," Monthly Notices of the Royal Astronomical Society, vol. 252, p. 300, 1991.

[5] P. A. Crowther and L. J. Smith, "An infrared spectral analysis of two Cygnus WNE stars," Astronomy and Astrophysics, vol. 305, no. 2, pp. 541-550, 1996.

[6] J. C. Mauerhan, S. D. Van Dyk, and P. W. Morris, "12 new galactic wolf-rayet stars identified via 2MASS + Spitzer/ GLIMPSE," Publications of the Astronomical Society of the Pacific, vol. 121, no. 880, pp. 591-605, 2009.

[7] M. F. Skrutskie, R. M. Cutri, R. Stiening et al., "The two Micron All Sky Survey (2MASS)," Astronomical Journal, vol. 131, no. 2, pp. 1163-1183, 2006.

[8] A. Moorwood, J.-G. Cuby, and C. Lidman, "SOFI sees first light at the NTT," Msngr, vol. 91, p. 9, 1998.

[9] L. J. Hadfield, S. D. Van Dyk, P. W. Morris, J. D. Smith, A. P. Marston, and D. E. Peterson, "Searching for hidden WolfRayet stars in the Galactic plane-15 new Wolf-Rayet stars," Monthly Notices of the Royal Astronomical Society, vol. 376, no. 1, pp. 248-262, 2007.

[10] A. Roman-Lopes, "SOAR-OSIRIS observations of the Sh 2-307 $\mathrm{H}$ ii region: triggered star formation beyond the Solar Circle," Monthly Notices of the Royal Astronomical Society, vol. 398, no. 3, pp. 1368-1382, 2009.

[11] D. F. Figer, I. S. Mclean, and F. Najarro, "A k-band spectral atlas of wolf-rayet stars," Astrophysical Journal, vol. 486, no. 1, pp. 420-434, 1997.

[12] P. A. Crowther, L. J. Hadfield, J. S. Clark, I. Negueruela, and W. D. Vacca, "A census of the Wolf-Rayet content in Westerlund 1 from near-infrared imaging and spectroseopy," Monthly Notices of the Royal Astronomical Society, vol. 372, no. 3, pp. 1407-1424, 2006.

[13] R. Indebetouw, J. S. Mathis, B. L. Babler et al., "The wavelength dependence of interstellar extinction from 1.25 to $8.0 \mu \mathrm{m}$ using glimpse data," Astrophysical Journal, vol. 619, no. 2, pp. 931-938, 2005.

[14] C. M. Dutra, E. Bica, J. Soares, and B. Barbuy, "New infrared star clusters in the southern Milky Way with 2MASS," Astronomy and Astrophysics, vol. 400, no. 2, pp. 533-539, 2003.

[15] E. P. Mercer, D. P. Clemens, M. R. Meade et al., "New star clusters discovered in the GLIMPSE survey," Astrophysical Journal, vol. 635, no. 1, pp. 560-569, 2005. 
[16] M. Kronberger, P. Teutsch, B. Alessi et al., "New galactic open cluster candidates from DSS and 2MASS imagery," Astronomy and Astrophysics, vol. 447, no. 3, pp. 921-928, 2006.

[17] N. Peretto and G. A. Fuller, "The initial conditions of stellar protocluster formation," Astronomy and Astrophysics, vol. 505, no. 1, pp. 405-415, 2009. 

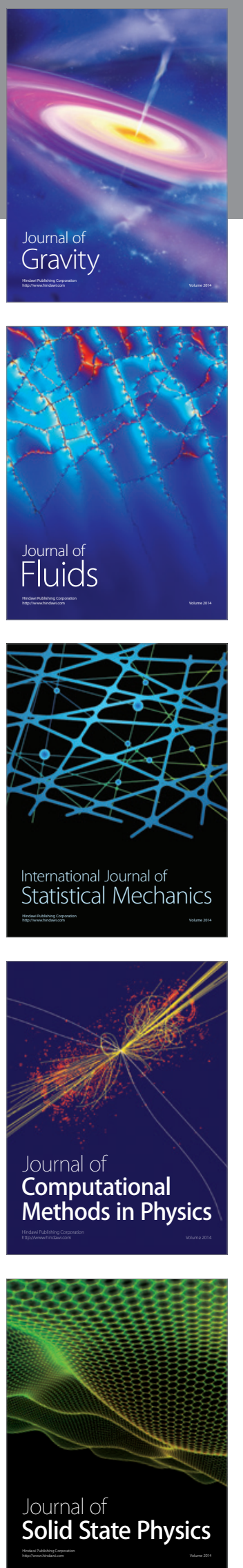

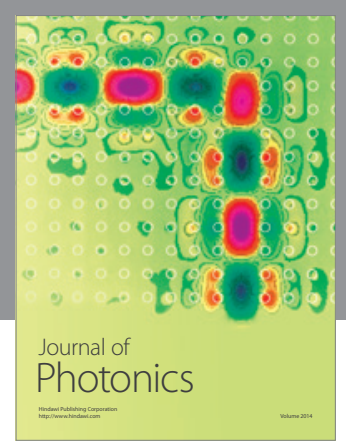

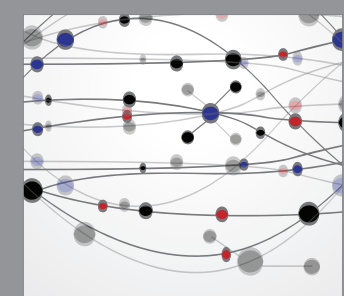

The Scientific World Journal
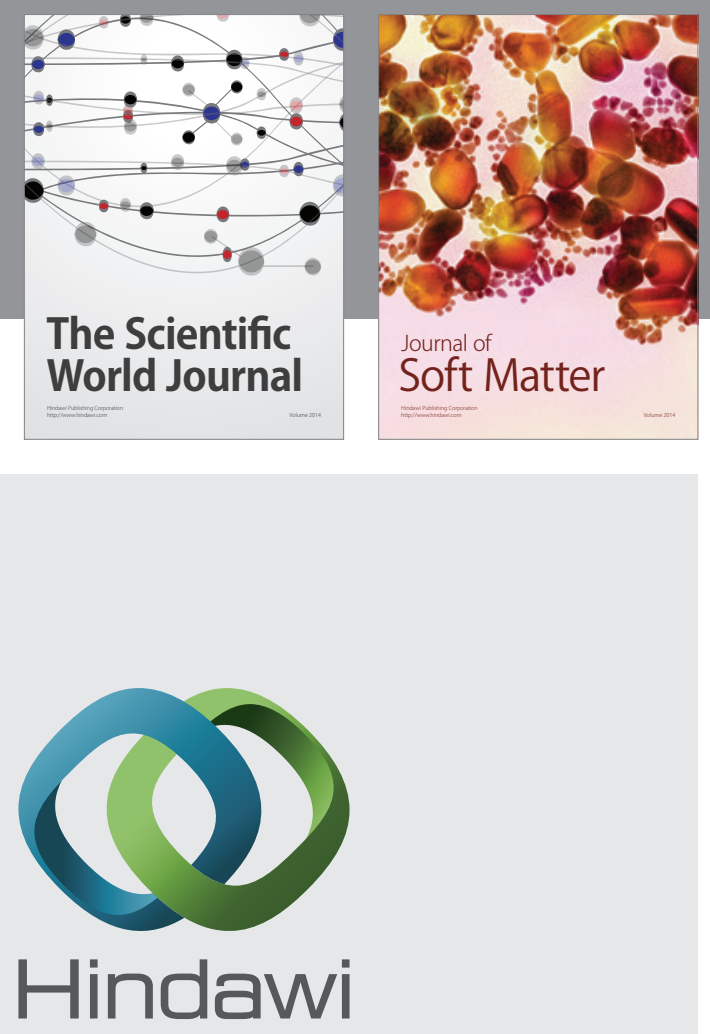

Submit your manuscripts at

http://www.hindawi.com
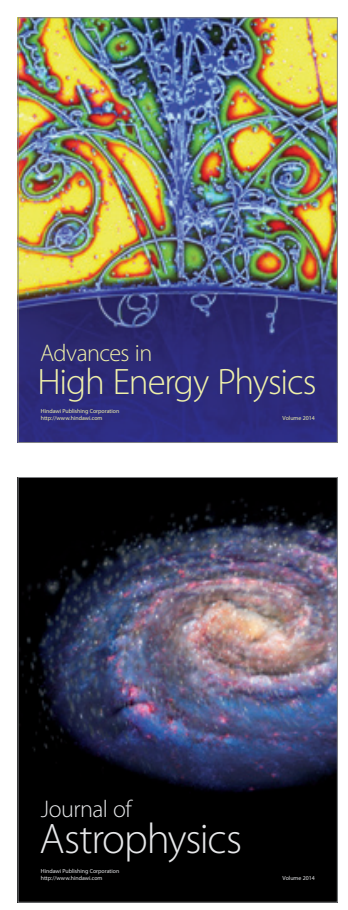
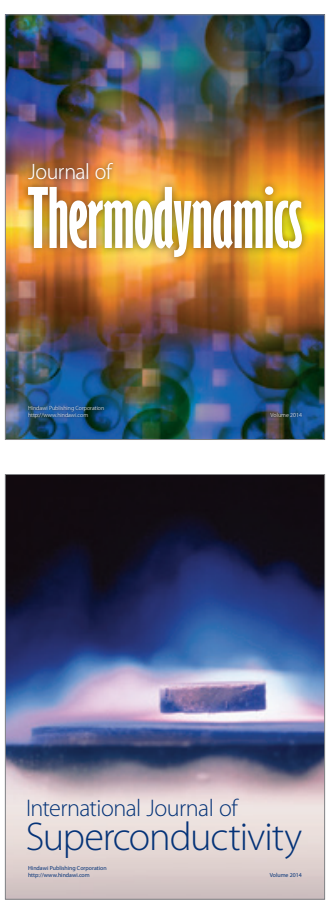
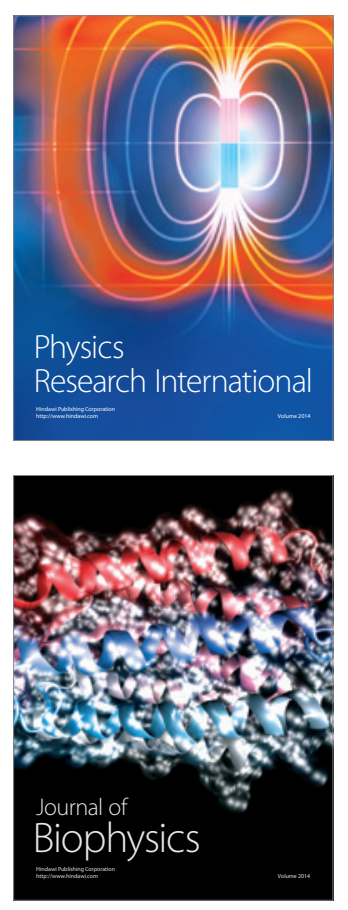
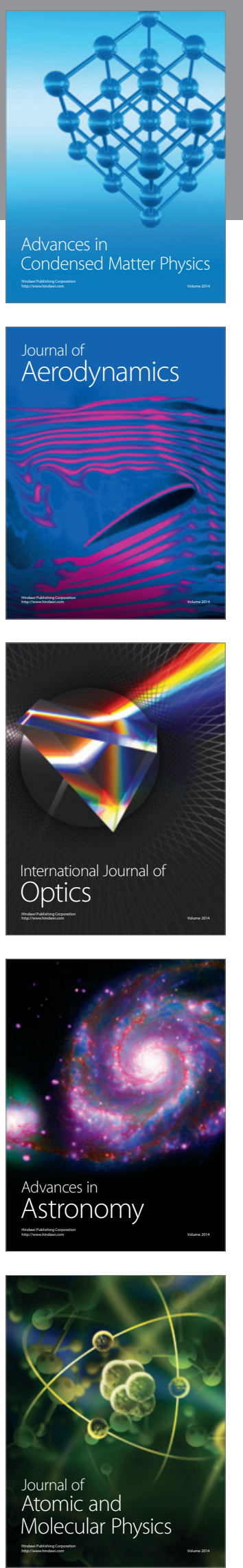